\title{
Preparation of a nano- and micro-fibrous decellularized scaffold seeded with autologous mesenchymal stem cells for inguinal hernia repair
}

This article was published in the following Dove Press journal:

International Journal of Nanomedicine

21 February 2017

Number of times this article has been viewed

\author{
Yinlong Zhangl,* \\ Yuanyuan Zhou',* \\ Xu Zhou ${ }^{2, *}$ \\ Bin Zhaol,* \\ Jie Chai' \\ Hongyi Liu' \\ Yifei Zheng' \\ Jinling Wang ${ }^{3}$ \\ Yaozong Wang ${ }^{4}$ \\ Yilin Zhao ${ }^{2}$ \\ 'Medical College, Xiamen University, \\ ${ }^{2}$ Department of Oncology and \\ Vascular Intervention Radiology, \\ ${ }^{3}$ Department of Emergency, \\ ${ }^{4}$ Department of Orthopaedics, \\ Zhongshan Hospital, Xiamen \\ University, Xiamen, People's Republic \\ of China
}

*These authors contributed equally to this work
Correspondence: Yilin Zhao Department of Oncology and Vascular Intervention Radiology, Zhongshan Hospital, Xiamen University, No 20I-209, Hubin South Road, 36I004 Xiamen, People's Republic of China Email zyllbz@gmail.com

\begin{abstract}
Prosthetic meshes used for hernioplasty are usually complicated with chronic pain due to avascular fibrotic scar or mesh shrinkage. In this study, we developed a tissue-engineered mesh (TEM) by seeding autologous bone marrow-derived mesenchymal stem cells onto nanosized fibers decellularized aorta (DA). DA was achieved by decellularizing the aorta sample sequentially with physical, mechanical, biological enzymatic digestion, and chemical detergent processes. The tertiary structure of DA was constituted with micro-, submicro-, and nanosized fibers, and the original strength of fresh aorta was retained. Inguinal hernia rabbit models were treated with TEMs or acellular meshes (AMs). After implantation, TEM-treated rabbit models showed no hernia recurrence, whereas AM-treated animals displayed bulges in inguinal area. At harvest, TEMs were thicker, have less adhesion, and have stronger mechanical strength compared to AMs $(P<0.05)$. Moreover, TEM showed better cell infiltration, tissue regeneration, and neovascularization $(P<0.05)$. Therefore, these cell-seeded DAs with nanosized fibers have potential for use in inguinal hernioplasty.
\end{abstract}

Keywords: nanobiomaterial, tissue engineering, inguinal hernia, hernioplasty, decellularized aorta

\section{Background}

Defects in collagen metabolism are one of the reasons for inguinal abdominal wall weakness, which usually leads to inguinal hernia. ${ }^{1}$ Hernia formation and recurrence are associated with altered collagen metabolism manifested by a decrease in the ratio of type I collagen to type III collagen. ${ }^{1}$ Tension-free inguinal hernia repair, which involves the placement of a mesh to strengthen the inguinal region rather than putting tension on muscles, has numerous advantages over herniorrhaphy. Therefore, it is the preferred method ever since it was established by Lichtensteina., ${ }^{2,3}$

Prosthetic meshes - such as knitted polypropylene, polytetrafluoroethylene, polytetrafluoroethylene (PTFE) plus hyaluronic acid, polyester, and polyglycolic acid - are widely used in hernia repair. ${ }^{4,5}$ Implantation of these nonabsorbable materials has been shown to restore abdominal wall integrity and reduce hernia recurrence. ${ }^{6}$ However, it was reported that patients who underwent hernioplasty with a synthetic mesh experience foreign body sensation, stiffness, and discomfort. ${ }^{7}$ Moreover, it has also been shown that prosthetic meshes cause severe inflammation, which leads to scar formation, chronic pain, viscera adhesions, bowel fistula, and mesh erosion into the bladder. ${ }^{8-11}$ Unlike native tissues, vascular ingrowths are prohibited in prosthetic grafts and it is difficult to get rid of bacteria once the graft is contaminated. As a result, prosthetic grafts 
should be avoided in patients with bacterial contamination and abdominal cavity infection. Moreover, when a surgical site infection occurs, it is imperative to remove the synthetic graft. ${ }^{8}$ Even without gross bacterial contamination, infected surgical incisions still occur in $\sim 5 \%$ patients. ${ }^{12}$

Recently, several extracellular matrices (ECMs) have been developed from naturally derived materials for the treatment of hernia and abdominal wall defects. ${ }^{13}$ These materials are easy for cell ingrowth, less susceptible to infection, and less likely to cause foreign body response. ${ }^{12}$ However, naturally derived materials tend to degrade and the mechanical strength and tensile strength decrease over time after implantation, ${ }^{14-16}$ which causes concern in clinical applications as tensile properties are necessities for grafts. Thus, it is important to understand not only the biological response to degradable biomaterials but also the mechanical properties of the implanted tissues over time. ${ }^{16}$ Also, this suggests that acellular collagen-based matrices alone may be insufficient scaffolds for abdominal wall reconstruction. ${ }^{16-18}$ The attenuation of graft strength over time is definitely a limitation of the acellular matrices that needs to be overcome. ${ }^{15}$

Earlier it was found that scaffolds seeded with cells exhibited stronger mechanical properties than the acellular matrices. In this study, we developed a tissue-engineered mesh (TEM) by seeding autologous mesenchymal stem cells (MSCs) into an organized nanofibrous ECM, which directs the growth, distribution, and function of MSCs for inguinal hernia repair in a rabbit model.

\section{Materials and methods}

All animal protocols were reviewed and approved by the Institutional Animal Care and Use Committee of Xiamen University. All animals were handled in accordance with the Animal Care Guidelines of the Science and Technology Department of Fujian Province.

\section{Decellularized aorta (DA) preparation}

Sheep aortas of $40 \mathrm{~mm}$ length and $15 \mathrm{~mm}$ internal lumen diameter were obtained from a local abattoir. The fresh aorta was dissected into subintima, media, and adventitia layer. The samples were rinsed in distilled water at $4^{\circ} \mathrm{C}$ for $24 \mathrm{~h}$ and then transferred to a shaker containing Triton-100 for $24 \mathrm{~h}$, and subsequently moved into a bottle containing $0.125 \%$ pepsin for $4 \mathrm{~h}$; they were then incubated with DNase and RNase for $4 \mathrm{~h}$, with the tissue weight:enzyme volume ratio adjusted to $1: 15$. The final product, DA, was freeze-dried and sterilized with ethylene oxide gas at room temperature.

\section{DNA quantification}

Native aorta and DA were cut into small pieces and placed in centrifuge tubes, which were later lyophilized. DNA was isolated using the TIANamp Genomic DNA kit (Tiangen, Beijing, People's Republic of China) according to the manufacturer's instructions. The amount of DNA was determined spectrophotometrically at $260 \mathrm{~nm}$.

\section{Mechanical testing of DA}

Strength tension was measured using a $1 \times 3 \mathrm{~cm}^{2}$ sample of DA. An Instron mechanical tester was used. Both ends of the sample were secured by clamps. Constant elongation ( $5 \mathrm{~mm} / \mathrm{min}$ ) was applied along the longitudinal axis of the patch until the samples were completely torn off. The maximal load was recorded.

\section{Isolation, proliferation, and purification of MSCs}

Bone marrow was harvested from the femurs of New Zealand White rabbits using previously described methods. ${ }^{19,20}$ Cells from the third passage were trypsinized, washed twice, and passed through a $10-\mu \mathrm{m}$ filter (Millipore) to collect the small-sized cells.

\section{Cell labeling}

The filtrated MSCs were labeled with the fluorescent dye PKH26 (Sigma-Aldrich, Shanghai, People's Republic of China) in accordance with a previous research. ${ }^{21}$ The labeling efficacy was determined by counting labeled cells under a fluorescence microscope (Eclipse E800, Nikon, Tokyo, Japan).

\section{Lactate dehydrogenase (LDH) assay}

LDH assay was performed using a commercial LDH kit (Beyotime, Jiangsu, People's Republic of China) according to previous report. ${ }^{22}$ Briefly, after each time point $(1,3,5$, and 7 days), $50 \mu \mathrm{L}$ of medium was collected from each well of the 24-well plate $(n=5)$ and transferred to a fresh 96-well plate. A total of $100 \mu \mathrm{L}$ of $\mathrm{LDH}$ assay mixture was added to each well and incubated in dark for $45 \mathrm{~min}$. To stop the reaction, $1 \mathrm{~N} \mathrm{HCl}$ (10\% volume) was added to each well. Absorbance values were recorded using a 96-well plate reader at $490 \mathrm{~nm}$.

\section{Rehydration analysis}

The dried DA samples with a mean weight of $3 \pm 0.1 \mathrm{~g}$ were immersed in phosphate-buffered saline (PBS) for $24 \mathrm{~h}$. Subsequently, samples were blotted with a piece of tissue 
to absorb the excess PBS on the surface and their weights were recorded.

\section{In vivo cellular infiltration}

DA samples $(2.5 \times 2.5 \mathrm{~mm})$ were implanted subcutaneously in C57BL/6 mice for cell infiltration analysis in vivo $(n=20)$. At days 3,14, and 30, the samples were harvested for histological examination $(n=5)$.

\section{Enzyme-linked immunosorbent assay (ELISA)}

The subcutaneously implanted samples were collected, weighed, washed, and homogenized in PBS containing 1\% penicillin and streptomycin $(100 \mu / \mathrm{mL})$ and then kept cool in cold, serum-free Dulbecco Modified Eagle Medium. After centrifugation, supernatants were collected and stored at $-20^{\circ} \mathrm{C}$ until analysis of the CXC chemokines macrophage inflammatory protein (MIP)-2 and keratinocyte chemoattractant (KC), which was conducted using the double-antibody Quantikine ELISA kits (R\&D Systems, Minneapolis, MN, USA) with recombinant murine KC and MIP-2 as standards.

\section{Cell seeding and maintenance in vitro}

Briefly, $1 \mathrm{~mL}$ of collagen I gel was mixed with $1 \mathrm{~mL}$ of Dulbecco Modified Eagle Medium. The labeled MSCs were suspended in a collagen-medium solution, and then were uniformly seeded onto and injected into the DA matrix which was placed in a plate. The cell density was $1 \times 10^{7}$ cell $/ \mathrm{mL}$. The seeding and injecting of MSCs into the matrix were performed 4 times in a week. After seeding, plates were placed in a $37^{\circ} \mathrm{C}$ incubator in an atmosphere containing $5 \% \mathrm{CO}_{2}$. To mimic bioreactor conditions, the medium was changed every $6 \mathrm{~h}$ during the culture period. Implants were routinely examined under light and fluorescence microscopes, and analyzed using scanning electron microscopy (SEM).

\section{Creation of the inguinal hernia model}

A total of 15 adult male rabbits (New Zealand White rabbits, approximate weight $2.5 \mathrm{~kg}$ ) were utilized in this study. The surgical procedures were performed under aseptic conditions. At the start of the experiment, the animals were anesthetized using an isoflurane $/ \mathrm{N}_{2} / \mathrm{O}_{2}$ inhalation. The abdomen was shaved and cleaned with $70 \%$ alcohol, after which a $4-\mathrm{cm}$ inguinal skin incision was made and skin flaps were raised. Alongside the spermatic cord, a defect between the skin and peritoneal cavity was made, with the inner part of the defect considered as the deep ring (inner ring) and the outer part as the superficial ring (outer ring). A hernia defect $(1.5 \times 2.5 \mathrm{~cm})$ alongside the spermatic cord was created by cutting through the full layers of the abdominal wall, including fascia and muscles, except peritoneum; the spermatic cord was at the center of this defect. This defect was finally sutured to create the hernia.

\section{Hernioplasty}

The rabbits were divided into 3 groups ( $n=5$ in each group). In the first group, the defect was repaired using TEM $(2.0 \times 2.5 \mathrm{~cm})$. In the second group, the defect was repaired using acellular mesh $(\mathrm{AM})(2.0 \times 2.5 \mathrm{~cm})$. Before transplantation, DA was rehydrated at room temperature for $15 \mathrm{~min}$ in sterile, $0.9 \%$ saline. The incisions were then closed over the mesh using sutures in the first two groups. In the third group, the animals were not treated with hernioplasty. The defect was closed only with the incision skin. Photographic records of the procedures were made. The skin sutures were removed 10 days after the operation. The defect was repaired around the spermatic cord. The inner and outer rings were then closed. The spermatic cord was in the middle of the implant.

\section{Animal care and reevaluation}

All animals were given 1 bolus of cefazolin $(0.5 \mathrm{mg})$ after the surgical procedure, and then housed in individual cages. They were weighed daily and checked for local and systemic complications, such as infection, wound dehiscence, signs of extreme pain, discomfort, and impaired mobility. The abdominal wall was checked by means of gross examination for hernia reoccurrence. The animals were checked and monitored two times a day.

\section{Gross examination of abdominal wall}

The animals were killed using high-dose ketamine 2 months after surgery. The abdominal wall was distended with $500 \mathrm{~mL}$ of saline to test for weakness. Any abnormal protrusion before or after testing was considered as a hernia.

\section{Examination for adhesions}

The inguinal area (including the entire repair site) was excised from the skin to the peritoneal cavity for examination. The exposure was performed gently to avoid breaking any adhesions. Photographs were taken throughout the process. Adhesion strength was graded semiquantitatively using an established grading scale. ${ }^{23}$ Grades ranged from 0 to 3 with increments of 0.5 : $0=$ no adhesions, $1=$ adhesions that could be easily freed with gentle tension, $2=$ adhesions that could be freed with blunt dissection, and $3=$ adhesions requiring sharp 
dissection to separate them from the implant site. Adhesions were graded by three independent and blinded observers.

\section{Mechanical testing}

To evaluate the strength of the border between the implant and native tissue, samples were harvested from similar locations in each animal at 2 months after implantation. Transverse sections from each animal were taken perpendicular to the implant-muscle interface; the sections included the implant, implant-muscle interface, and inguinal abdominal wall. All sutures were removed before mechanical testing, and all samples were assessed within $4 \mathrm{~h}$ of harvest and tested at room temperature. Samples were tested using the same method described in the section of Mechanical testing of DAs. The maximum load required to induce a complete break in the sample was recorded.

\section{Histological analysis}

Samples were fixed in 10\% neutral buffered formalin and embedded in paraffin. Sections were prepared for hematoxylin-eosin, Masson's trichrome, and Van Gieson's staining. With Van Gieson's, collagen stains red, and with Masson's trichrome, collagen stains green or blue. Slides were immunostained with antibodies against von Willebrand factor (vWF; Boster, Wuhan, People's Republic of China). A commercial immunohistochemical kit was used along with 3,3'-diaminobenzidine tetrahydrochloride as the enzyme substrate: brown granular deposits indicated the presence of vWF. The vessels per unit area $\left(1 \mathrm{~mm}^{2}\right)$ were counted in five randomly chosen fields per slide of each portion by three blinded pathologists. The average number of vessels in one portion was used for the assessment of vascular density.

\section{In vivo differentiation of MSCs}

The slides were examined for fluorescence to trace the implanted MSCs, and then incubated with vWF (Boster, Wuhan, People's Republic of China) antibody and fluorescein isothiocyanate-conjugated secondary antibody (Boster, Wuhan, People's Republic of China) to detect endothelial cells. The signals were visualized using a confocal laser scanning microscope (Leica).

\section{SEM}

Specimens were fixed in 1\% buffered glutaraldehyde and $0.1 \%$ buffered formaldehyde for 1 and $24 \mathrm{~h}$, respectively, and dehydrated using a graded ethanol series. The dried samples were mounted on an aluminum stub, coated with gold using a sputter coater, and examined under an SEM (Tescan, Brno, Czech Republic).

\section{Statistical analysis}

All data are expressed as mean \pm standard error of mean, and $n$ represents the number of animals per experimental group. Statistical comparisons between the groups were performed using the rank sum test. Differences were considered significant at $P<0.05$.

\section{Results \\ Tertiary structure of DA}

After decellularization of fresh aorta, cellular components were removed. The DA was left with a smooth intima (Figure 1A), and an organized structure consisted of collagen and elastin fibers (Figure 1A-I). The size of these fibers varied from microscale to nanoscale. The aortic samples were dissected into three layers as intima (subintima), media, and adventitia for further evaluation of its structure. The threedimensional DA had a tertiary structure and was composed of three kinds of fibers: microscale collagen fibers with diameter size ranging from 1 to $6 \mu \mathrm{m}$, submicroscale fibers with diameter size from 100 to $999 \mathrm{~nm}$, and nanoscale fibers with diameter size below $100 \mathrm{~nm}$. In the different layers of the DA, the amount of collagen and elastin fibers varied along with their thickness. In the subintima, which was mainly considered as basement membrane for endothelial cells tethering and adhesion, there were considerable amount of submicroscale and nanoscale fibers, and fewer microsized fibers (Figure 1B). SEM examination of the cross-section of DA showed that the scaffold had a multilayered and porous structure, and was mainly composed of microsized fibers (Figure 1C). The microsized fibers were parallel to each other, and interconnected by submicrosized collagen fibers and nanosized fibers to form the network (Figure 1D-F). The distance between the microfibers, submicrofibers, and nanofibers was $\sim 20 \mu \mathrm{m}, 5 \mu \mathrm{m}$, and $200 \mathrm{~nm}$, respectively. The average pore diameter was $19.4 \mu \mathrm{m}$. After further treatment with collagenase, the microsized fibers were removed of epitendon, and left with thousands of average-sized nanofibers (Figure $1 \mathrm{G}$ and $\mathrm{H}$ ). There were more and thinner nanosized fibers in the adventitia, while there were fewer and thicker fibers in the media layer (Figure 1I). The number of fibers in the adventitia was significantly higher than that in the subintima and media; the number in the subintima was significantly higher than that in the media. The DA was a 3D organized network which was well preserved after decellularization (Figure 1J). The fibers in the media were significantly thicker than those in the subintima and adventitia; the fibers in the subintima were slightly thicker than those in the adventitia (Figure $1 \mathrm{~K}$ and $\mathrm{L}$ ). 

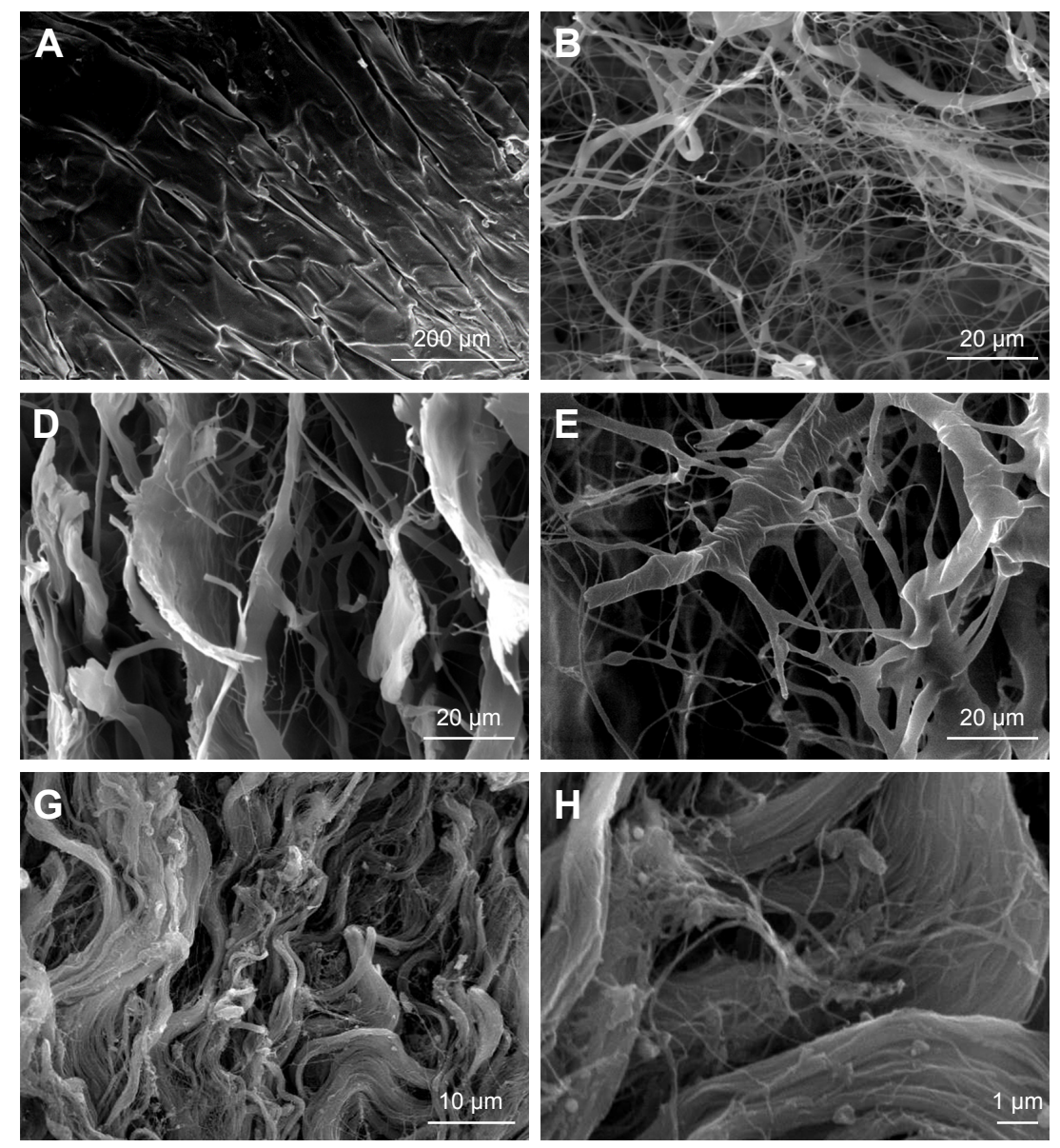

$\mathbf{J}$

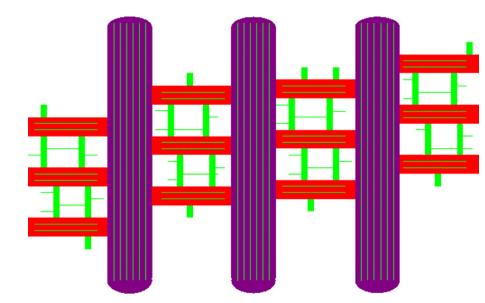

Microscale fiber $\left.\_\geq 1 \mu \mathrm{m}\right)$

- Submicroscale fiber $(<1 \mu \mathrm{m}, \geq 100 \mathrm{~nm})$

Nanoscale fiber $(<100 \mathrm{~nm})$
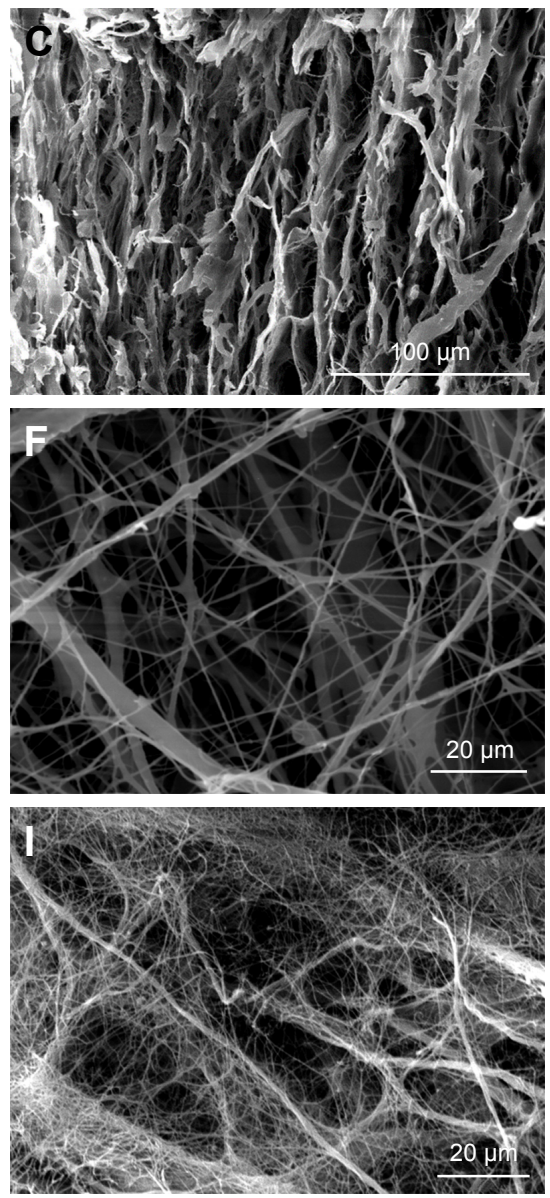

K
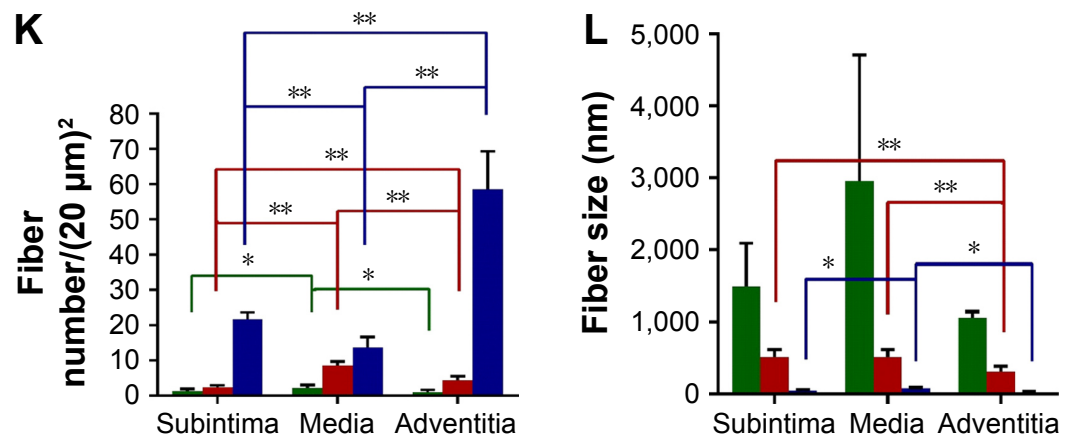

Microscale fiber Submicroscale fiber $\boldsymbol{n}$ Nanoscale fiber

Figure I Size distribution of collagen fibers in different layers of decellularized aorta.

Notes: (A) The intima was smooth and removed of cellular components (bar[b]: $200 \mu \mathrm{m})$. (B) The subintima mainly consisted of submicrosized fibers (b: $20 \mu \mathrm{m})$. (C) The cross-section was multilayered and removed of cellular components (b: $100 \mu \mathrm{m})$. (D) The media mainly consisted of microsized fibers (b: $20 \mu \mathrm{m})$. (E) The microsized fibers in media were interconnected with submicrosized and nanosized fibers (b: $20 \mu \mathrm{m})$. (F) The nanoscale fibers originated from microsized or submicrosized fibers in the media were as the bridge between submicrofibers (b: $20 \mu \mathrm{m}$ ). (G) After removing of epitenon, microsized fibers were composed of thousands of nanoscale fibers (b: $10 \mu \mathrm{m}$ ). (H) The nanoscale fibers within microsized fibers were averagely sized and regularly aligned (b: I $\mu \mathrm{m}$ ). (I) The adventitia mainly consisted of nanosized fibers (b: I0 $\mu \mathrm{m}$ ). (J) Structure diagram. (K) Quantification of microsized, submicrosized, and nanosized fibers in subintima, media, and adventitia ( $* P<0.05$ and $* * P<0.0$ ) . (L) Size distribution of microsized, submicrosized, and nanosized fibers in subintima, media, and adventitia $(* P<0.05$ and $* * P<0.01)$.

\section{Gross view and histological analysis}

DA was a soft, white ECM (Figure 2A). Cellular components had been removed completely from the fresh aorta (Figure 2B-D). Staining showed that the DA was mainly composed of collagen and elastin fibers (Figure 2C and D).

\section{Quantitative DNA analysis}

The Student's $t$-test for unpaired samples showed that the DNA quantification was significantly different $(P<0.05)$ between the samples obtained from the DA (16.19 $\pm 0.66 \mathrm{ng} / \mathrm{mg}$ of tissue) and the native tissues $(277.81 \pm 15.10 \mathrm{ng} / \mathrm{mg}$; Figure 2E). 

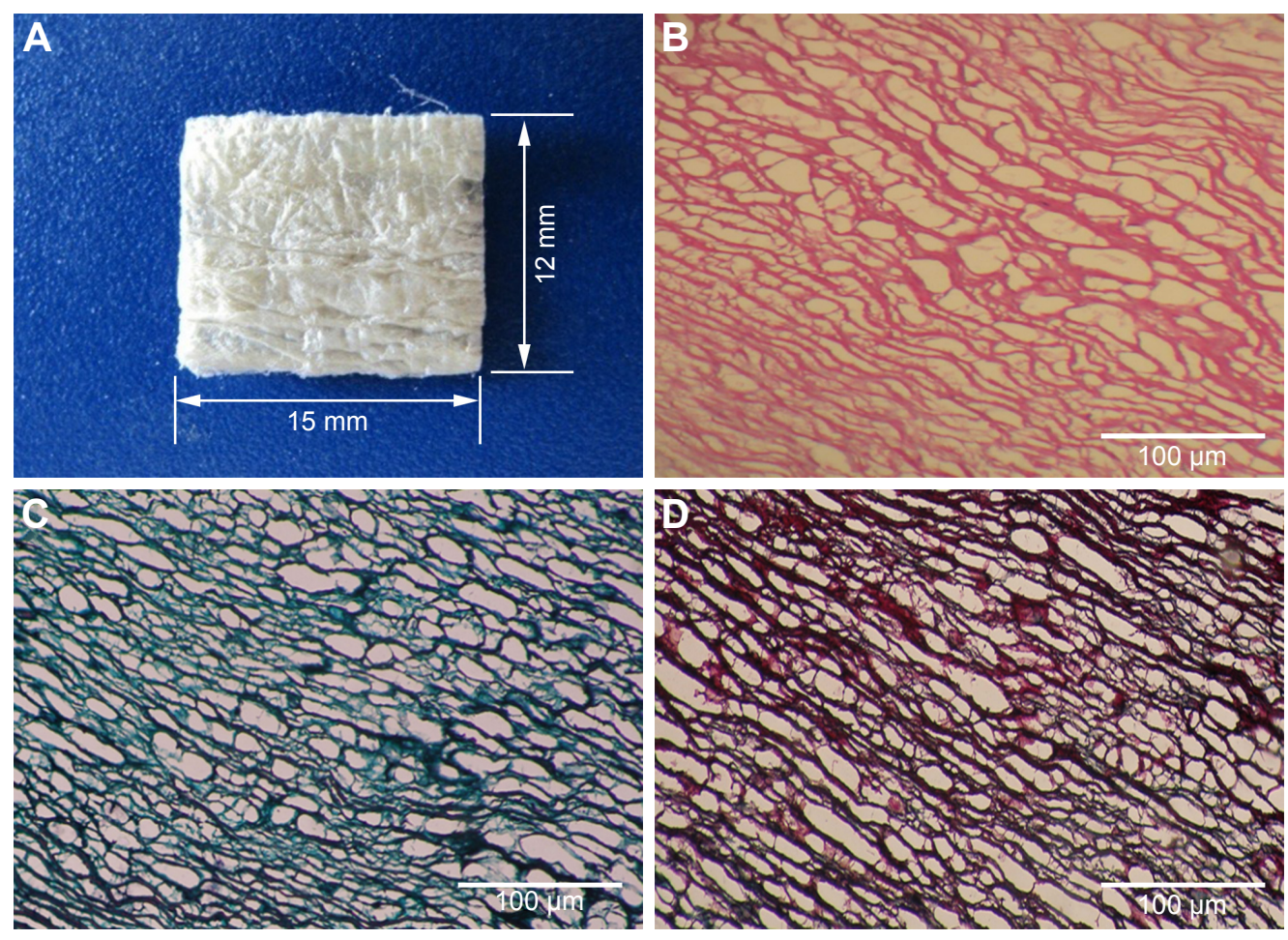

E
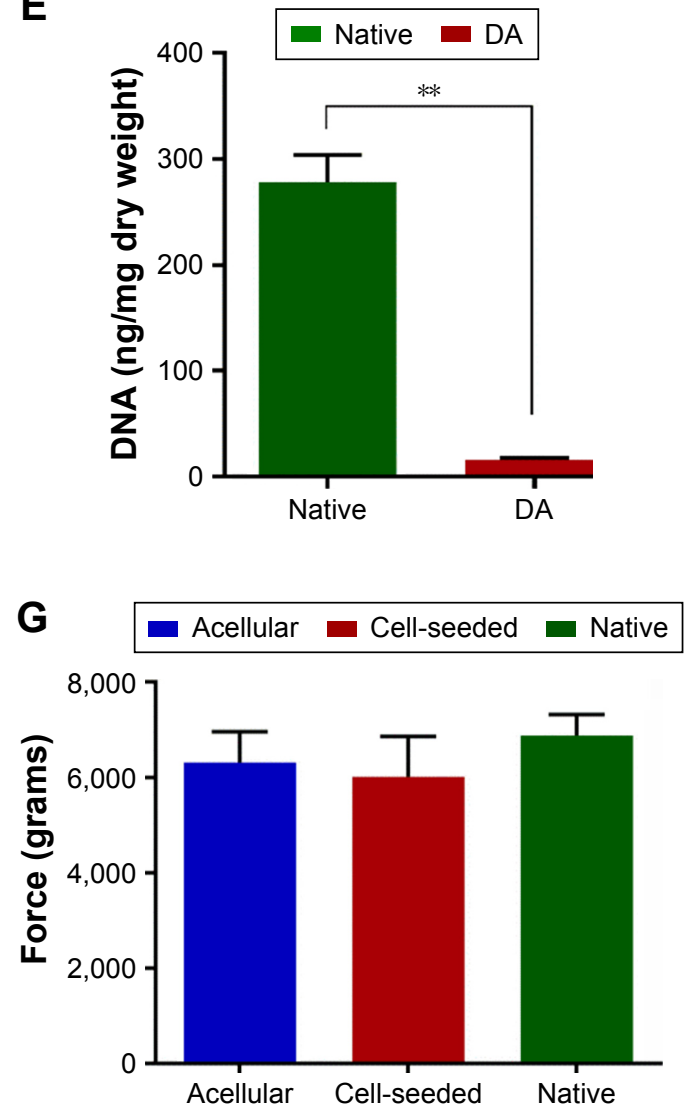

$\mathbf{F}$
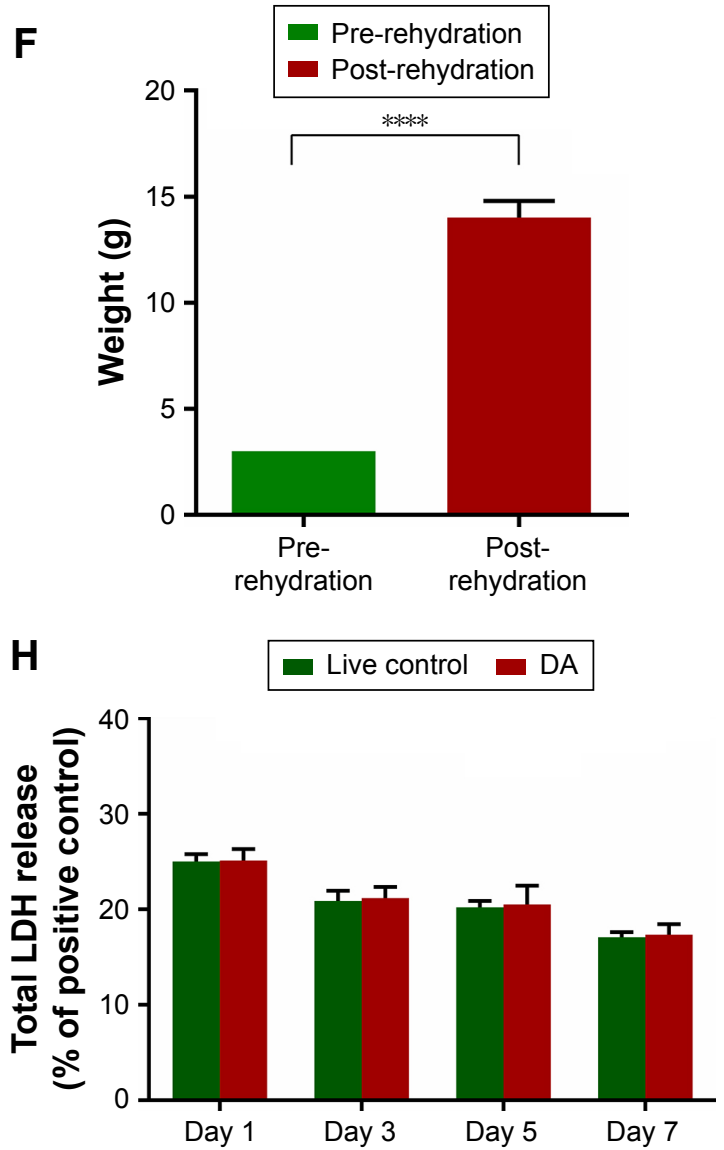

Figure 2 Characterization of decellularized aorta (DA)

Notes: (A) Gross view of DA. (B) DA was multilayered and was totally removed of cellular components (hematoxylin and eosin, bar [b]: I00 $\mu$ m). (C) Collagen layers in Masson's trichrome staining (green color; b: $100 \mu \mathrm{m}$ ). (D) Collagen layers in Van Gieson's staining (red color; b: I00 $\mu \mathrm{m}$ ). (E) Amount of DNA in control and DA. Bars represent mean \pm SEM $(* * P<0.05, n=3)$ compared with the native. $(\mathbf{F})$ Rehydration test $(* * * * P<0.000 I, n=5)$. (G) Suture strength between the acellular meshes, cell-seeded mesh, and fresh aorta (native) $(P>0.05)$. (H) Lactate dehydrogenase (LDH) assays for checking the cytotoxicity of DA $(P>0.05)$. 


\section{Rehydration analysis}

The rehydration analysis indicated that dry DA could rehydrate $4.02 \pm 0.87$ times over its own weight (Figure $2 \mathrm{~F}$ ).

\section{Strength test}

The strength of the DA was slightly lower than that of the fresh aorta $(P>0.05$; Figure $2 \mathrm{G})$. Strength retention of the scaffold was measured to determine whether the grafts could withstand the pressure forces of intra-abdominal cavity, which ranges from 1 to $6.5 \mathrm{mmHg}$ in a normal human being. ${ }^{24,25}$ The strength of the DA was different - but not to a significant degree - from that of the native tissue, which can withstand pressures of about 2,000 mmHg, ${ }^{19}$ suggesting that the scaffold had enough strength to withstand abdominal forces.

\section{LDH assay}

Figure $2 \mathrm{H}$ demonstrated the total $\mathrm{LDH}$ released from MSCs after 1, 3, 5, and 7 days of culture on DA scaffolds. There was no significant difference between the LDH released from MSCs on DA scaffolds and the LDH released from control cells $(P>0.05)$.

\section{In vivo host immunological reaction against DA}

We found that after implantation, the cells gradually infiltrated into the decellularized scaffold (Figure 3G-I). Once the mesh was implanted, the levels of MIP-2 and $\mathrm{KC}$ increased during the initial 14 days, after which they decreased gradually (Figure $3 \mathrm{~J}$ ). Thus, an immunological reaction occurred at an early stage, while at 30 days, the inflammation largely subsided.

\section{Proliferation and infiltration of MSCs}

It took about 4 weeks to harvest enough seeding cells. DAs were seeded with small-sized MSCs and cultured for 14 days. Cells began to grow and proliferate within the matrix after seeding. The seeded cells adhered well in the scaffold and covered the matrix within 5 days (Figure 3A). A high density of cells was present within the DA at 14 days (Figure 3B). The labeled MSCs were seeded and proliferated on the scaffold (Figure 3C) and within the scaffold (Figure 3D). SEM showed that the MSCs proliferated, grew well, and uniformly spread on the scaffold (Figure 3E), whereas the MSCs inside the scaffold grew well along with collagen
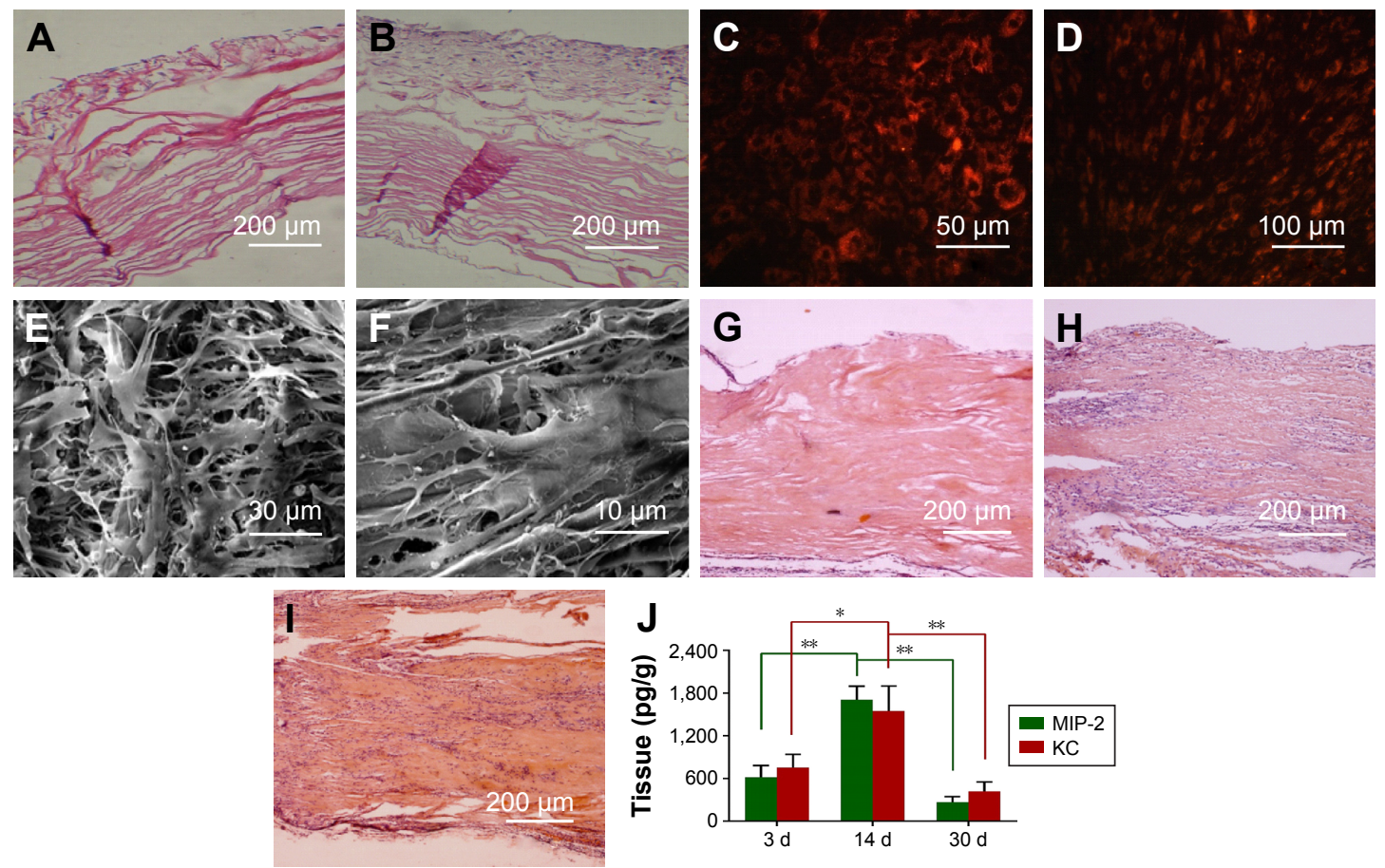

Figure 3 Recellularization of decellularized aorta (DA) and inflammation in vivo.

Notes: (A) One week after seeding of mesenchymal stem cells (MSCs; hematoxylin and eosin [H\&E]; bar [b]: $200 \mu \mathrm{m})$. (B) Two weeks after MSCs seeding (H\&E; b: $200 \mu \mathrm{m})$. (C) Labeled MSCs on the intima (b: $50 \mu \mathrm{m}$ ). (D) Labeled MSCs within the scaffolds were aligned in the same direction (b: I00 $\mu \mathrm{m})$. (E) MSCs grew irregularly on the scaffold at 7 days (scanning electron microscope [SEM]; b: $30 \mu \mathrm{m}$ ). (F) MSCs were aligned along collagen fibers and in regular morphologies (SEM; b: I0 $\mu \mathrm{m}$ ). (G) Cellular infiltration in vivo at 3 days (H\&E; b: $200 \mu \mathrm{m})$. (H) Cellular infiltration in vivo at 14 days (H\&E; b: $200 \mu \mathrm{m})$. (I) Cellular infiltration in vivo at 28 days (H\&E; b: $200 \mu \mathrm{m})$. (J) The level of macrophage inflammatory protein (MIP)-2 and $K C(* P<0.05$ and $* * P<0.01, n=5)$.

Abbreviation: KC, keratinocyte chemoattractant. 
fibers or between the fibers within the matrix, aligned in a specific direction (Figure 3F).

\section{Transplantation of recellularized hernia grafts}

Inguinal hernia models were successfully built in 15 male rabbits (Figure 4A-C). The hernia disappeared when the site of the inner ring was pressed, and the hernia appeared again when the pressure was removed (Figure 4D-F). In the first group, the TEMs were sutured around the spermatic cord to repair the defect in the rabbits that donated the bone marrow ( $n=5$; Figure $4 \mathrm{G}$ ). In the second group, the AMs were sutured around the spermatic cord to repair the defects $(n=5)$. In the third group, the defects were closed and covered only with skin $(n=5)$. To check for hernia recurrence, the animals were periodically examined for abdominal bulge after implantation. The third group (defect closed only with the skin) died within 10 days because of the incarceration of the intestine. Abdominal bulge was not noted in the other two groups before the harvest. No animals experienced incision or implantation infections.

\section{Hernia recurrence}

Before infusion of $500 \mathrm{~mL}$ of saline into the abdominal cavity, the TEM and AM groups showed no hernia. After abdominal enlargement, no hernia was found in the TEM animals (Figure 4H), whereas all the rabbits in the AM group showed implant protrusion and hernia (Figure 4I).

\section{Characterization of the implants}

Fewer adhesions were observed between the implants and the skin in the TEM group, whereas obvious adhesion was observed in the AM group. The TEM implants were opaque and thick (Figure 4J), whereas the AMs were semitransparent, thin, and in a bulge (Figure $4 \mathrm{~K}$ ). The borderline between $\mathrm{AM}$ and native tissues could be recognized (Figure 4L).
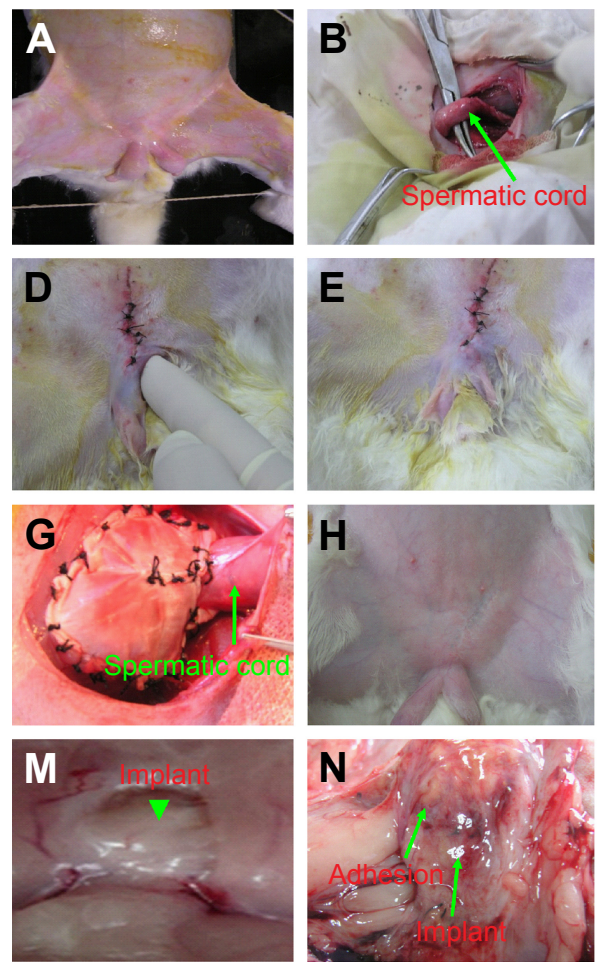
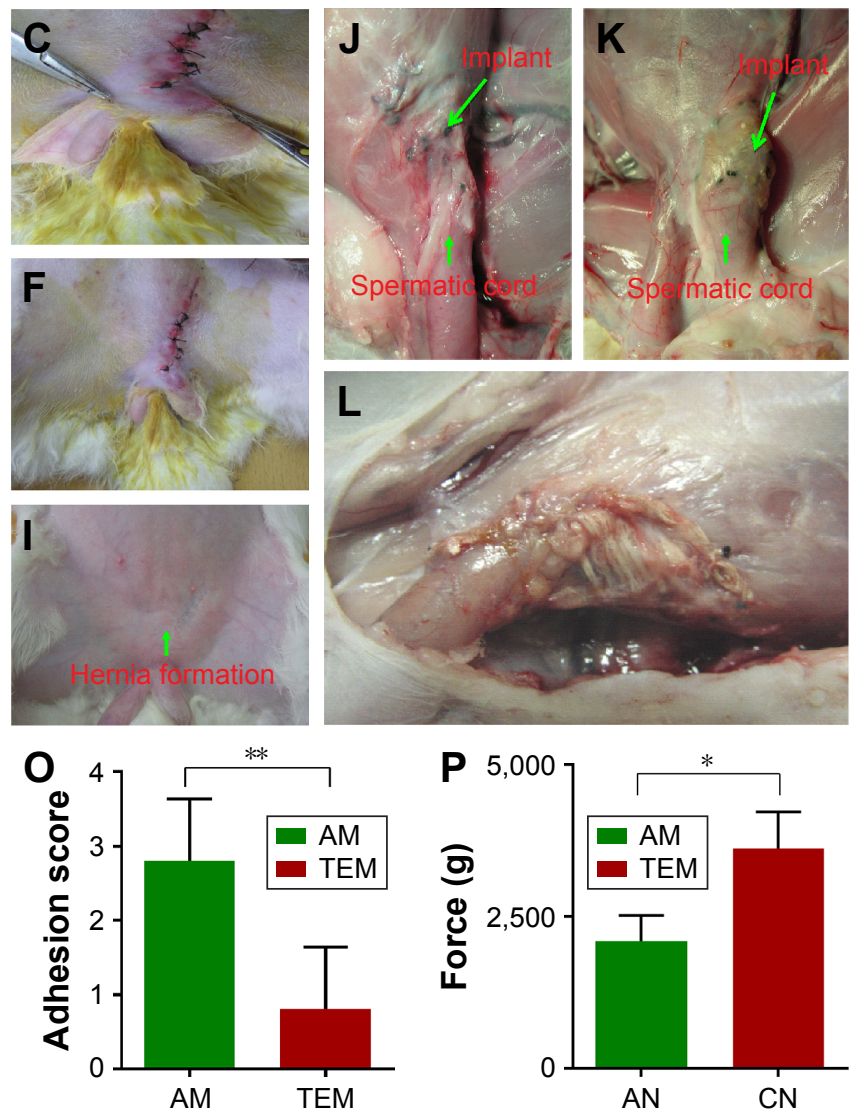

Figure 4 Hernia model, hernioplasty, and gross examination after transplantation.

Notes: (A) Normal groin. (B) Spermatic cord. (C) The right testicle was much larger than the left, which was considered as an inguinal hernia; the content within the hernia sac was intestine. (D) The content in the testicle was pushed back to the abdominal cavity. (E) The hernia disappeared. (F) After pressure was removed, the intestine returned back to the testicle. (G) The inguinal hernia was repaired. (H) No hernia formation in tissue-engineered mesh (TEM) group. (I) A bulge (arrow) was found in acellular meshes (AMs) group. (J) Absence of adhesion in peritoneum of TEM and well-formed blood vessels were at the border of TEM and native tissue. (K) Severe adhesion in AM. (L) No hernia formation was observed in TEM, and the implants were oblique and thick. (M) Hernia formation in AM group. (N) The AMs were transparent and thin. (O) Adhesion scores $(* * P<0.01, n=5)$. (P) The strength of the border between the implants and native muscle $(* P<0.05, n=5)$. 
The thickness of the TEM was similar to the neighboring native tissues. In each case, the TEM was thicker than the AM. It was difficult to find adhesions between the peritoneum and peritoneal contents in the TEM group (Figure 4M). Adhesions were severe in the AM group (Figure $4 \mathrm{~N}$ ). The mean adhesion score was $0.25 \pm 0.5$ in the TEM group compared with 2.25 \pm 1 in the AM group ( $P<0.01$; Figure 4O). At 60 days after implantation, the breaking strength of the interface between TEM and host tissue was much higher than that of the AM $(P<0.05$; Figure 4P).

\section{Histological analysis of the implants}

TEM regenerated very well with cellular infiltration; minor scar formation was noted at the border (Figure 5A). The interface between TEM and native tissue grew closely and firmly (Figure 5C). Tissue regeneration and remodeling were observed in TEMs. It was difficult to find unabsorbed scaffold in TEM implants (Figure 5C). Special staining showed the presence of collagen in the TEMs and at the border between the TEM and the native tissue (Figure 5E); moreover, collagen structure in TEMs was similar to that in native tissue. However, in AMs, the border was not firmly closed (Figure 5B); there was lesser cell infiltration and poorer tissue regeneration in AMs, and a considerable amount of unabsorbed scaffold could be found in AMs (Figure 5D). Masson's trichrome staining showed that irregular and poor collagen formation was observed in AM (Figure 5F).

\section{Tracing the seeded cells in the matrix}

Two months after implantation, the labeled MSCs were found in TEMs (Figure 5G). Staining for vWF showed capillary formation in TEMs (Figure 5G). Some MSCs differentiated into endothelial cells and formed capillaries (Figure 5G). Fluorescence intensity was reduced, probably due to migration of MSCs and dilution of the dye incorporated into the cell membrane as a result of cell division. The TEMs showed intense staining for vWF, which indicated good vascular formation. In contrast, fewer vascular formations were observed in AMs. Capillary number in TEMs was significantly higher than that in AMs ( $87 \pm 9$ vs $62 \pm 13$ vessels $/ \mathrm{mm}^{2}$, $P<0.05$; Figure 5H).

\section{SEM analysis}

SEM confirmed good regeneration of the peritoneum in TEMs (Figure 5I and J), similar to that in native endothelium.
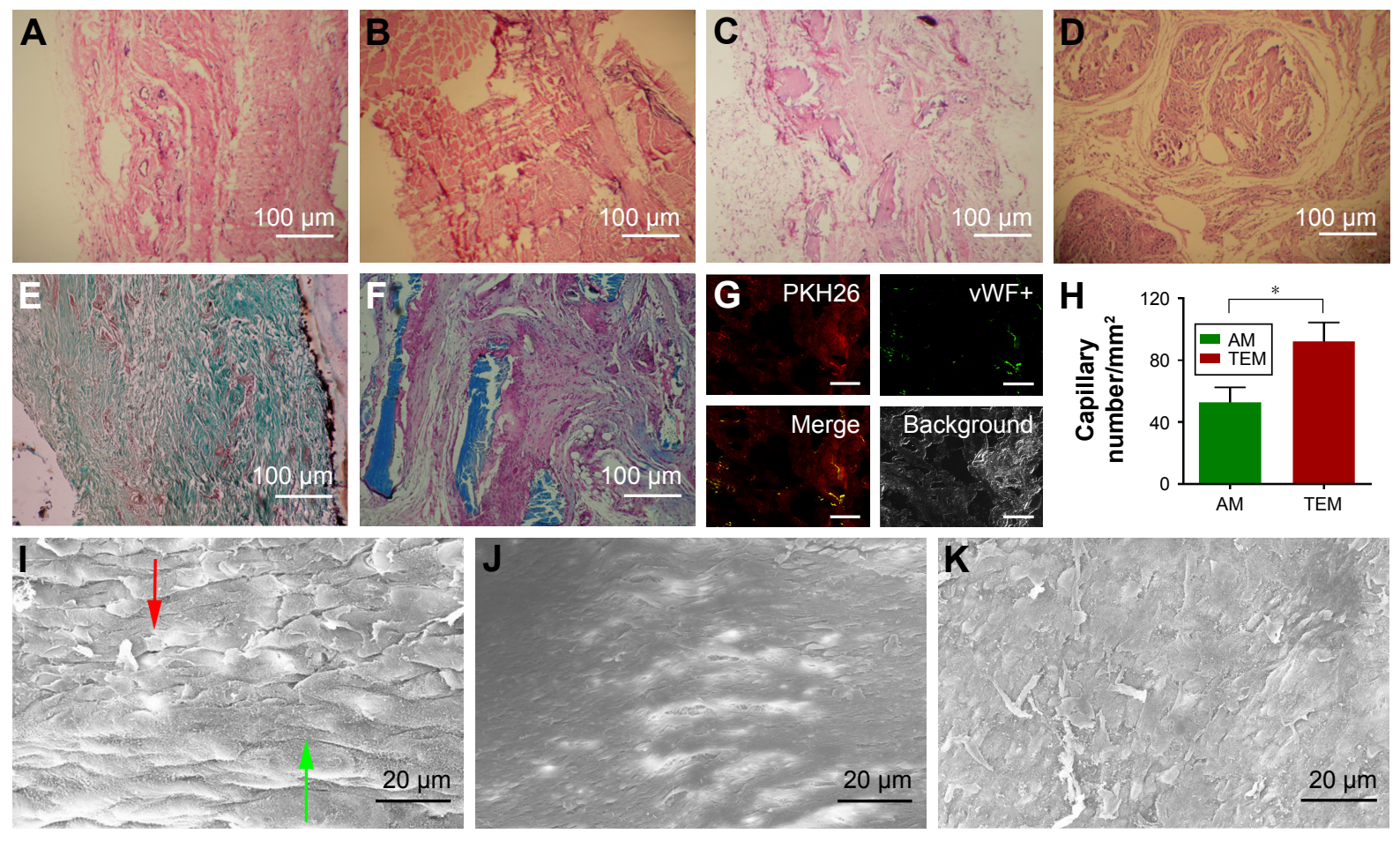

Figure 5 Histological analyses 2 months after hernioplasty.

Notes: (A) Tissue-engineered mesh (TEM; hematoxylin and eosin [H\&E]; bar [b]: $100 \mu \mathrm{m}$ ). (B) Acellular meshes (AMs; H\&E; b: $100 \mu \mathrm{m})$. (C) Interface between native muscle and TEM (H\&E; b: $100 \mu \mathrm{m})$. (D) Interface between native tissue and AM (H\&E; b: $100 \mu \mathrm{m})$. (E) Border between native muscle and TEM was close and firm (Masson's trichrome staining; b: $100 \mu \mathrm{m}$ ). (F) Border between the native muscle and AM (Masson's trichrome staining; b: $100 \mu \mathrm{m}$ ). (G) Colocalization of MSCs (red) and von Willebrand factor ( $\mathrm{vWF}$; green; b: $100 \mu \mathrm{m})$. (H) Capillary density $(* \mathrm{P}<0.05, \mathrm{n}=5)$. (I) Border between native peritoneum (red arrow) and the peritoneum (green arrow) adjacent to TEM (scanning electron microscope [SEM]; b: $20 \mu \mathrm{m}$ ). (J) Peritoneum in TEM (SEM; b: $20 \mu \mathrm{m}$ ). (K) Rough peritoneum in AM (SEM; b: $20 \mu \mathrm{m})$.

Abbreviation: MSCs, mesenchymal stem cells. 
SEM revealed that poor peritoneum formation was observed in the AM (Figure 5K).

\section{Discussion}

In this research, we developed a purely natural, uniform, and highly organized nano- and microscale fibrous decellularized scaffold derived from blood vessel with tertiary structure for the application of hernia tissue engineering. We also achieved efficient in vitro recellularization inside the grafts, and the viability of MSCs was maintained. The scaffold exerted excellent capabilities in directing and supporting MSCs alignment and proliferation along the underlying fibers. Finally, we demonstrated that the feasibility of transplanting these tissue-engineered hernia grafts for inguinal hernia repair, and their ability to facilitate tissue regeneration in inguinal abdominal defect. Our data also indicated that inguinal hernia repaired with a natural nanomaterial mesh engineered with MSCs leads to improved mechanical and functional outcomes when compared with an equivalent AM.

Improvements in nanotechnology and nanobiomaterials in decades have been used extensively in promoting tissue engineering and regenerative medicine. ${ }^{26-28}$ Nanobiomaterials have been considered better candidates than the traditional tissue engineering scaffolds because these nanoscale biomaterials can effectively mimic the characters of natural tissues, in which cells directly contact their surrounding nanoscale ECM, which exerts a key role in supplying mechanical support, directing cell adhesion and growth, as well as regulating tissue development, homeostasis, and regeneration. ${ }^{29,30}$ In the interest of simulating more chemical and biological components of the natural ECM, various electrospun nanoscale scaffolds have been fabricated to imitate the topography of natural ECM for tissue engineering and regenerative medicine. ${ }^{30,31}$ However, most of these nanometer-grade scaffolds are prepared with synthetic materials or natural polymer blends, which are not able to adequately simulate the complex morphology and composition of natural ECM. Although current electrospunning techniques can regulate the mechanical and chemical properties of the nanometer fibers, only natural ECM can potentially possess more appropriate nanoscale topography, mechanical support, and chemical and biological promoting agents for cellular activities at the same time. ${ }^{32}$ Although investigators have developed different types of decellularized animal-derived scaffolds for tissue engineering, none of them presented an originally organized nanometer-level biomaterial.

The preparation of DA involves a multistep process to remove both the epi-aorta's cells and the cells inside the tissue without destroying the main structure, and leaves a residual matrix that preserved original ECM collagen fiber structure in native tissues which keep original morphology, biochemical constituents, and mechanical properties. ${ }^{31}$ The matrices were composed of three levels of micro-, submicro-, and nanosized fibers that retained the topography and morphology of the naturally aortic ECM. DAs were composed of microsized ( $\geq 1 \mu \mathrm{m})$, submicrosized $(<1 \mu \mathrm{m}$ and $\geq 100 \mathrm{~nm}$ ), and nanosized collagen fibers $(<100 \mathrm{~nm})$ in a tertiary structure. The sizes of the collagen fibers at different layers are quite different. Among them, the microscale fibers maintained the mechanical property of the original aortic tissue, the submicrosized fibers provided the sites for cells adherence and tethering, whereas the nanoscale collagen fibers were for the MSCs spreading, stretching, and proliferating. ${ }^{33}$ They also provide the desired geometry and porosity for MSCs without the limitation of poor cell penetration. MSCs can perceive the topography of the underlying substrate and respond to the physical stimuli by adjusting their alignment and migration. After MSCs being seeded, the DA fibers were closely packed together and glued by adhesive molecules, which possess multiple binding domains capable of binding collagen and proteoglycans, as well as the cell surface. ${ }^{34}$

It was reported that small-sized MSCs undergo rapid self-renewal and have a greater potential for multipotent differentiation than larger cells. ${ }^{35}$ The engraftment of MSCs demonstrates site-specific and multipotent differentiation, and they are present at sites of wound healing and tissue regeneration after transplantation. ${ }^{36} \mathrm{We}$ found that some of the grafted MSCs had differentiated into endothelial cells and formed capillaries. With the development of blood supply within the implants, the new tissue gains more tensile strength from the regenerated cells and tissue than that in AMs. This means that the tensile strength of the constructed scaffold depended not only on the mechanical properties of the biomaterial but also on the regenerated tissue. ${ }^{37,38}$

The performance of the MSCs-seeded constructs in the abdominal surgical animal model demonstrates its efficacy in preventing adhesion formation with living cell surfaces. The seeded MSCs that highly resembled native peritoneum acted as a continuous monolayer over the entire peritoneal tissue surface and protected organs against adhesion.

\section{Acknowledgments}

We would like to thank Jianyin Zhou and Yun Liu for advice on creation of the inguinal hernia model and Xuan Zhu for 
the SEM technical assistance. This work was supported by the Project of National Natural Science Foundation of China (No 81371698).

\section{Author contributions}

Y Zhang, Y Zhou, XZ, BZ, JC, HL, Y Zheng, JW, and YW collected and analyzed the data. Y Zhao designed the research and wrote the manuscript. All authors contributed toward data analysis, drafting and revising the paper and agree to be accountable for all aspects of the work.

\section{Disclosure}

The authors report no conflicts of interest in this work.

\section{References}

1. Henriksen NA, Yadete DH, Sorensen LT, Agren MS, Jorgensen LN. Connective tissue alteration in abdominal wall hernia. Br J Surg. 2011;98(2):210-219.

2. Lichtenstein IL, Shulman AG. Ambulatory outpatient hernia surgery. Including a new concept, introducing tension-free repair. Int Surg. 1986;71(1):1-4.

3. Nathan JD, Pappas TN. Inguinal hernia: an old condition with new solutions. Ann Surg. 2003;238(Suppl 6):S148-S157.

4. LeD, Deveney CW, Reaven NL, Funk SE, McGaughey KJ, Martindale RG. Mesh choice in ventral hernia repair: so many choices, so little time. Am J Surg. 2013;205(5):602-607.

5. Kugel RD. Minimally invasive, nonlaparoscopic, preperitoneal, and sutureless, inguinal herniorrhaphy. Am J Surg. 1999;178(4):298-302.

6. Chastan P. Tension-free inguinal hernia repair: a retrospective study of 3000 cases in one center. Int Surg. 2005;90(1):48-52.

7. Klinge U, Klosterhalfen B, Müller M, Schumpelick V. Foreign body reaction to meshes used for the repair of abdominal wall hernias. Eur J Surg. 1999;165(7):665-673.

8. Paton BL, Novitsky YW, Zerey M, Sing RF, Kercher KW, Heniford BT. Management of infections of polytetrafluoroethylene-based mesh. Surg Infect (Larchmt). 2007;8(3):337-341.

9. Paily A, Thornton M. Chronic pain following a Lichtenstein inguinal hernia repair: a clinical and legal dilemma. ANZ J Surg. 2009;79(7-8):517-520.

10. Tolino MJ, Tripoloni DE, Ratto R, Garcia MI. Infections associated with prosthetic repairs of abdominal wall hernias: pathology, management and results. Hernia. 2009;13(6):631-637.

11. Di Vita G, Milano S, Frazzetta M, et al. Tension-free hernia repair is associated with an increase in inflammatory response markers against the mesh. Am J Surg. 2000;180(3):203-207.

12. Milburn ML, Holton LH, Chung TL, et al. Acellular dermal matrix compared with synthetic implant material for repair of ventral hernia in the setting of peri-operative Staphylococcus aureus implant contamination: a rabbit model. Surg Infect (Larchmt). 2008;9(4):433-442.

13. Diaz JJ Jr, Conquest AM, Ferzoco SJ, et al. Multi-institutional experience using human acellular dermal matrix for ventral hernia repair in a compromised surgical field. Arch Surg. 2009;144(3):209-215.

14. Hutmacher DW, Goh JC, Teoh SH. An introduction to biodegradable materials for tissue engineering applications. Ann Acad Med Singapore. 2001;30(2):183-191.

15. Meintjes J, Yan S, Zhou L, Zheng S, Zheng M. Synthetic, biological and composite scaffolds for abdominal wall reconstruction. Expert Rev Med Devices. 2011;8(2):275-288.
16. Zhao Y, Zhang Z, Wang J, et al. Abdominal hernia repair with a decellularized dermal scaffold seeded with autologous bone marrow-derived mesenchymal stem cells. Artif Organs. 2012;36(3):247-255.

17. Lai JY, Chang PY, Lin JN. Body wall repair using small intestinal submucosa seeded with cells. J Pediatr Surg. 2003;38(12):1752-1755.

18. Fauza DO, Marler JJ, Koka R, Forse RA, Mayer JE, Vacanti JP. Fetal tissue engineering: diaphragmatic replacement. J Pediatr Surg. 2001;36(1):146-151.

19. Zhao Y, Zhang S, Zhou J, et al. The development of a tissue-engineered artery using decellularized scaffold and autologous ovine mesenchymal stem cells. Biomaterials. 2010;31(2):296-307.

20. Colter DC, Sekiya I, Prockop DJ. Identification of a subpopulation of rapidly self-renewing and multipotential adult stem cells in colonies of human marrow stromal cells. Proc Natl Acad Sci U S A. 2001; 98(14):7841-7845.

21. Chen J, Wang C, Lü S, et al. In vivo chondrogenesis of adult bonemarrow-derived autologous mesenchymal stem cells. Cell Tissue Res. 2005;319(3):429-438.

22. Lalwani G, Gopalan A, D'Agati M, et al. Porous three-dimensional carbon nanotube scaffolds for tissue engineering. J Biomed Mater Res A. 2015;103(10):3212-3225.

23. Butler CE, Navarro FA, Orgill DP. Reduction of abdominal adhesions using composite collagen-GAG implants for ventral hernia repair. J Biomed Mater Res. 2001;58(1):75-80.

24. De Waele JJ, De Laet I, Malbrain ML. Rational intraabdominal pressure monitoring: how to do it? Acta Clin Belg. 2007;62(Suppl 1):16-25.

25. Malbrain ML. Different techniques to measure intra-abdominal pressure (IAP): time for a critical re-appraisal. Intensive Care Med. 2004;30(3):357-371.

26. Zhang L, Webster TJ. Nanotechnology and nanomaterials: Promises for improved tissue regeneration. Nano Today. 2009;4(1):66-80.

27. Peng S, Jin G, Li L, et al. Multi-functional electrospun nanofibres for advances in tissue regeneration, energy conversion \& storage, and water treatment. Chem Soc Rev. 2016;45(5):1225-1241.

28. Cross LM, Thakur A, Jalili NA, Detamore M, Gaharwar AK. Nanoengineered biomaterials for repair and regeneration of orthopedic tissue interfaces. Acta Biomater. 2016;42:2-17.

29. Swinehart IT, Badylak SF. Extracellular matrix bioscaffolds in tissue remodeling and morphogenesis. Dev Dyn. 2016;245(3):351-360.

30. Frantz C, Stewart KM, Weaver VM. The extracellular matrix at a glance. J Cell Sci. 2010;123(Pt 24):4195-4200.

31. Schaefer L, Schaefer RM. Proteoglycans: from structural compounds to signaling molecules. Cell Tissue Res. 2010;339(1):237-246.

32. Seras-Franzoso J, Tatkiewicz WI, Vazquez E, et al. Integrating mechanical and biological control of cell proliferation through bioinspired multieffector materials. Nanomedicine (Lond). 2015;10(5):873-891.

33. Baker BM, Trappmann B, Wang WY, et al. Cell-mediated fibre recruitment drives extracellular matrix mechanosensing in engineered fibrillar microenvironments. Nat Mater. 2015;14(12):1262-1268.

34. Theocharis AD, Skandalis SS, Gialeli C, Karamanos NK. Extracellular matrix structure. Adv Drug Deliv Rev. 2016;97:4-27.

35. Samsonraj RM, Rai B, Sathiyanathan P, et al. Establishing criteria for human mesenchymal stem cell potency. Stem Cells. 2015;33(6): $1878-1891$.

36. Liechty KW, MacKenzie TC, Shaaban AF, et al. Human mesenchymal stem cells engraft and demonstrate site-specific differentiation after in utero transplantation in sheep. Nat Med. 2000;6(11):1282-1286.

37. Liu W, Thomopoulos S, Xia Y. Electrospun nanofibers for regenerative medicine. Adv Healthc Mater. 2012;1(1):10-25.

38. Song Z, Peng Z, Liu Z, Yang J, Tang R, Gu Y. Reconstruction of abdominal wall musculofascial defects with small intestinal submucosa scaffolds seeded with tenocytes in rats. Tissue Eng Part A. 2013;19(13-14): $1543-1553$. 


\section{Publish your work in this journal}

The International Journal of Nanomedicine is an international, peerreviewed journal focusing on the application of nanotechnology in diagnostics, therapeutics, and drug delivery systems throughout the biomedical field. This journal is indexed on PubMed Central, MedLine, CAS, SciSearch $\AA$, Current Contents $\AA /$ Clinical Medicine,

Journal Citation Reports/Science Edition, EMBase, Scopus and the Elsevier Bibliographic databases. The manuscript management system is completely online and includes a very quick and fair peer-review system, which is all easy to use. Visit http://www.dovepress.com/ testimonials.php to read real quotes from published authors.

Submit your manuscript here: http://www.dovepress.com/international-journal-of-nanomedicine-journal 